\title{
The Effects of Baduanjin Exercise on BMI, Waist-Hip Ratio and Lipids Among Perimenopausal Women
}

\author{
Li-Yun $\mathrm{Szu}^{1} \&$ Lee-Ing Tsao ${ }^{1}$ \\ ${ }^{1}$ Doctoral Candidate, Department of Nursing, National Taipei University of Nursing and Health Sciences, Taipei, \\ Taiwan \\ Correspondence: Li-Yun Szu, Adjunct Professor, Department of Nursing, National Taipei University of Nursing \\ and Health Sciences, No.365, Ming-te Road, Poitou District, Taipei City, Taiwan. E-mail: \\ leeing.ntunhs@gmail.com
}

Received: February 4, 2020

Accepted: February 21, 2020

Online Published: March 2, 2020

doi:10.20849/ijsn.v5i1.716

URL: https://doi.org/10.20849/ijsn.v5i1.716

\begin{abstract}
Background: The Prevalence of metabolic syndrome is increasing in perimenopausal women. BMI, waist-hip ratio (WHR) and lipids are important factors for controlling metabolic syndrome. The Baduanjin exercise is a traditional Chinese exercise that promotes the integration of physical and mental health, and it is easy to practice at home and fit for busy perimenopausal women. However, the health effects needed to be evaluated.

Aim: The purpose of this quasi-experimental research was to evaluate the effects, after twelve weeks of practicing Baduanjin exercise.

Methods: This study was conducted at two Buddhism painting clubs, using a parallel-design, control trial recruiting perimenopausal women. There were three instruments: (1) BMI, (2) Waist-Hip Ratio, (3) Lipids-HDL and LDL. The intervention effects from the study baseline to a twelve weeks follow-up were estimated using T-test to evaluate the effectiveness of Baduanjin exercise. The Baduanjin exercise intervention included one-on-one teaching and telephone follow-up to help women practice Baduanjin exercise of 10-15 minutes twice per day for twelve weeks at home. Result: A total of 54 women aged 40 to 60 were recruited from two Buddhism painting clubs. Twenty-seven women were in the experimental group, while 27 women were in the control group. Positive effects were found after 12 weeks doing Baduanjin exercise of 10-15 minutes twice per day at home. In the experimental group, women improved their health by significantly decreasing BMI, increasing HDL, decreasing LDL and more slowly increasing WHR compared with the control group. Conclusion: Baduanjin exercise is a good home exercise to suggest perimenopausal women to improve their BMI, waist-hip ratio and lipid
\end{abstract}

Keywords: Baduanjin, Low-Density Lipoprotein (LDL), High-Density Lipoprotein (HDL), perimenopausal women

\section{Introduction}

\subsection{Perimenopaual Is High Risk Factor for Cardiovascular Disease}

The metabolic syndrome (MetS) was more prevalent among postmenopausal women than among premenopausal women. After menopause, as estrogen levels decrease, health risks begin to increase, including chronic diseases such as high blood sugar, high blood pressure, and high blood lipids. Risk factors for cardiovascular disease (CVD) are higher than women of childbearing age. Appropriate lifestyle changes can prevent from CVD such as healthy diet, quitting smoking, regular exercise and maintaining proper weight (Baghbani-Oskouei, Tohidi, Asgari, Ramezankhani, Azizi \& Hadaegh, 2018; Colpani, et al., 2018; Ford, Zhao, Tsai \& Li, 2011; Gurka, Vishnu, Santen \& DeBoer, 2016; Hashemi Nazari, Shakiba , Khalili, Hadaegh, Tohidi \& Azizi, 2015; Hunter, Hardy, Norton \& Griffiths, 2016; Palacios et al., 2010; Mc Sweeney et al., 2016; Muka et al., 2016; Mumusoglu \& Yildiz, 2019; Nejat et al., 2014; Tabatabaei-Malazy et al., 2018). Several studies have shown that in postmenopausal women "excess waist circumference or abdominal obesity", "overweight and obese" hypertension, dyslipidemia, hyperglycemia, and high abdominal obesity rates are closely related to the prevention and treatment of CVD and health promotion. (Cengiz, Kaya, Suzen Caypinar \& Alay, 2019; Colpani, et al., 2018; Korsager Larsen M, Matchkov, 2016; Lin, \& Lee, 2018; Ministry of Health and Welfare, 2019 a, b, c; 
Pal, Radavelli-Bagatini \& Ho, 2013; Wang, Qin \& Cui, 2016).

\subsection{Explore Importance of the Baduanjin and Perimenopause}

The Baduanjin is a complete fitness exercise originated in the Song Dynasty and has a history of more than 1,000 years. It is a set of traditional self-care functions based on the principles of traditional Chinese medicine. This is one of the traditional Chinese qigong exercises. It is easy to learn, soft and slow, and easy to exercise regularly (Wikipedia, 2019; Wan \& Zhang, 2015). It includes eight steps of step-by-step exercise, involving dynamics and physiology theory, to balance the body through simple exercises with natural energy. The coordination of breathing, energy, and thought is a gentle and safe aerobic exercise, which focuses on regulating breathing to achieve physical and mental balance. (Li, Wu, Jin \& Pan, 2019). Practitioners of Baduanjin exercise concentrate on adjusting breathing to bring about balance of the body and mind, and studies indicate that this regular physical exercise provides benefits regarding the psychological rehabilitation. Baduanjin is easy to learn, soft, and has the characteristics of slow stretching. It does not feel uncomfortable during the exercise, and it is not limited by equipment and venue. It is a simple, convenient, and non-injury exercise that can be voluntarily performed at home, which is conducive to the patient's continuous regularity motion. It is a sports therapy worth promoting in the community. Existing literature showed that it can promote physical and mental health (Li et al., 2014, Li et al., 2015; Li, Ge, Liu, Zhang, Wang, Si \& Zhai, 2019; Li, Wu, Jin \& Pan 2019; Xiao, \& Zhuang, 2015; Xiao, Wang, Gu, Cai \& Ma, 2018; Ying et al., 2019).

The menopause age of Chinese women is about 49-51 years old, the average life expectancy of women is 84-85 years, and one third of life are after menopause. Therefore, the maintenance of menopausal health is very important. However, menopausal women are busy with the burden of various roles, lack of motivation is limited to the location or movement of the exercise, and it is difficult to perform the exercise regularly. Therefore, if we develop a sport that can be exercised at home, simpl and without sports injuries, we should increase the regular willingness of menopausal women to prevent various chronic diseases such as cardiovascular disease and hyperlipidemia, and achieve the goal of promoting menopausal women's health. Li et al. (2014) found that the satisfaction with Baduanjin exercise was $100 \%$, indicating that the Baduanjin exercise has high compliance with the subjects.

Therefore, there is a simple and easy-to-learn menopause campaign at home, which is very important for the health care of Chinese menopausal women. However, there is a lack of evidence for regular implementation of the Baduanjin exercise on LDL, HDL, WHR, and BMI among menopausal women. The purpose of this study is to evaluate the effectiveness of Baduanjin exercise intervention on community menopausal women's BMI, WHR, LDL, and HDL.

\section{Method}

\subsection{Research Design and Subjects}

This study was conducted at two Buddhism painting clubs using a parallel-design, control trial recruiting menopausal women. There were three instruments: (1) BMI, (2) Waist-Hip Ratio (3) Lipids - HDL and LDL. The two painting clubs are 2.7 miles apart; women in each group lived far enough apart that the control group would not be contaminated by the experimental group or vice versa. The intervention effects from the study baseline to a twelve-week follow-up were estimated using T-test to evaluate the effectiveness of Baduanjin exercise. The Baduanjin exercise intervention included one-on-one teaching and telephone follow-up to help women practice Baduanjin exercise of 10-15 minutes twice per day for twelve weeks at home. Both the experimental and control groups were pre-tested with three measurements. Subsequently, Baduanjin exercise intervention was implemented for the experiment group. Twelve weeks later both groups were post-tested using the same three measurements and compared on changed scores. An application for permission to conduct this study was approved. A written informed consent was obtained from each woman prior to enrollment in the study. All participants knew that they were going to have a test 12 weeks later. They were also informed that they were volunteer participants in the study and they had the right to refuse to participate and the right to withdraw at any time without jeopardy.

\subsection{Identify Subsections}

It is both conventional and expedient to divide the Method section into labeled subsections. These usually include a section with descriptions of the participants or subjects and a section describing the procedures used in the study. The latter section often includes description of (a) any experimental manipulations or interventions used and how they were delivered-for example, any mechanical apparatus used to deliver them; (b) sampling procedures and sample size and precision; (c) measurement approaches (including the psychometric properties of 
the instruments used); and (d) the research design. If the design of the study is complex or the stimuli require detailed description, additional subsections or subheadings to divide the subsections may be warranted to help readers find specific information.

Include in these subsections the information essential to comprehend and replicate the study. Insufficient detail leaves the reader with questions; too much detail burdens the reader with irrelevant information. Consider using appendices and/or a supplemental website for more detailed information.

\subsection{Experimental Manipulations or Interventions}

A standard intervention protocol with the same intensity was designed and used across all sits. The WHR and BMI data were measured by using body composition analyzer - InBody 2.0; LDL and HDL were measured by drawing blood. The recruiting criteria were as following: (1) women were aged 40-60 years who had irregular exercises; (2) were able to walk freely; (3) had no neuromuscular problems to restrict their activities; (4) unstable health conditions such as cancer, severe cardiopulmonary, liver, and renal dysfunction were ruled out.

\section{Measures and Covariates}

Include in the Method section information that provides definitions of all primary and secondary outcome measures and covariates, including measures collected but not included in this report. Describe the methods used to collect data (e.g., written questionnaires, interviews, observations) as well as methods used to enhance the quality of the measurements (e.g., the training and reliability of assessors or the use of multiple observations). Provide information on instruments used, including their psychometric and biometric properties and evidence of cultural validity.

\subsection{Research Design}

The researcher individually taught the exercises and precautions of Baduanjin. The participant women in the experimental group were required to be familiar with the intensive training of the Baduanjin steps 2-3 times one week before beginning of the research. The women were taught to understand and experience the feelings of Baduanjin's qi, blood, and meridians. Then twice a day, in the morning and the evening, a 10-15 minutes of Baduanjin exercise were performed for 12 weeks. During the 12 weeks, the researcher asked the women about the implementation status and difficulties encountered through the phone, meeting the women once a week in Buddhism painting class to check performing the Baduanjin exercise before class. At this time, the researcher evaluated the correctness and execution of the Baduanjin exercises of the research subjects.

The exercise intensity applied reached 11-14 in the Rating of Perceived Exertion (RPE), which means that the feeling of exercise is "easy", "it's a little hard" exercise intensity. The Rating of Perceived Exertion (RPE) was used to evaluate the physical and psychological sensory intensity of exercise after the Baduanjin exercise performed. RPE is a scale developed by Swedish physiologist Gunnar Borg (1982). After Borg tests, the conscious exercise intensity and the measured exercise intensity are highly correlated with their physiological indicators (Borg, 1982). A simple number from the "very, very easy" to "very, very strenuous" index is used to represent the subjective perceived exercise intensity. Studies have found that RPE can accurately reflect the intensity change during exercise, and it is related to the number of heartbeats during exercise. There was a significant positive correlation between physiological variables such as oxygen uptake (Rago, Brito, Figueiredo, Costa, Krustrup, \& Rebelo, 2019).

In addition, for ethical reasons the women in the control group received a copy of the exercise brochure at the end of the study instead of the beginning of the study. It provided women in the control group as self-education material.

\subsection{Data Analysis:}

A Chi-square test and $t$ test were used to determine the difference between the two groups of women with BMI, WHR, LDL, HDL and their demographic data.

Differences within groups were analyzed using paired t-tests and differences between groups were analyzed using t-tests. A level of $\mathrm{P}<0.05$ indicated statistical significance. The study used SPSS 12.0 for statistical analysis.

\section{Results}

\subsection{The Homogenous Comparison of Two Groups}

At the beginning and post-test (after 12 weeks of intervention), 60 women were recruited, 6 women withdrew because of household relocation or personal issues. There were no significant differences of menstrual status and 
other baseline

characteristics between women who dropped out and the women who participated the whole process. The full participation rate of this study was $91 \%$. There were 27 participants in the experimental group; while there were 27 women in the control group.

participants in the experimental group; while there were 27 women in the control group. Table 1 gave the baseline characteristics of participants in the two groups. There were no significant differences in the demographic data, such as educational background, age, marital status, vocation, ministration status, drugs usage, personal past history, diet pattern and WHR, BMI, HDL and LDL in pretest. (See Table 1).

Table 1. Demographic data and pretest $(N=54)$

\begin{tabular}{|c|c|c|c|c|}
\hline Items & Experimental $(\mathrm{n}=27)$ & Control $(n=27)$ & $\mathrm{X}^{2}$ or $\mathrm{t}$ & $\mathrm{P}$ \\
\hline & $\%$ or mean $( \pm \mathrm{SD})$ & $\%$ or mean $( \pm \mathrm{SD})$ & & \\
\hline Age & $46.2(5.2)$ & $48.5(6.5)$ & -1.396 & 0.169 \\
\hline Educational & & & 2.148 & 0.342 \\
\hline College and above & $16(59.3)$ & $11(40.7)$ & & \\
\hline Middle & $8(29.6)$ & $10(37.0)$ & & \\
\hline Elementary & $3(11.1)$ & $6(22.2)$ & & \\
\hline Marital status & & & 3.000 & 0.083 \\
\hline Married & $15(55.6)$ & $21(77.8)$ & & \\
\hline Single & $12(44.4)$ & $6(22.2)$ & & \\
\hline Vocation & & & 4.800 & 0.091 \\
\hline Retired/housewife & $6(22.2)$ & $12(44.4)$ & & \\
\hline Part-time & $2(7.4)$ & $4(14.8)$ & & \\
\hline Employed & $19(70.4)$ & $11(40.7)$ & & \\
\hline Menstrual status & & & 3.703 & 0.157 \\
\hline postmenopause & $6(22.2)$ & $9(33.3)$ & & \\
\hline perimenopause & $5(18.5)$ & $9(33.3)$ & & \\
\hline premenopause & $16(59.3)$ & $9(33.3)$ & & \\
\hline Medication & & & 0.164 & 0.685 \\
\hline none & $23(82.1)$ & $24(88.9)$ & & \\
\hline yes & $4(14.8)$ & $3(11.1)$ & & \\
\hline Chronic disease & & & 1.421 & 0.233 \\
\hline none & $21(77.8)$ & $17(63.0)$ & & \\
\hline yes & $6(22.2)$ & $10(37.0)$ & & \\
\hline Vegetarian & & & 0.355 & 0.551 \\
\hline no & $9(33.3)$ & $7(25.9)$ & & \\
\hline yes & $18(66.7)$ & $20(74.1 \%)$ & & \\
\hline WHR & $0.86(0.42)$ & $0.85(0.04)$ & 0.70 & 0.95 \\
\hline BMI & $22.19(2.52)$ & $21.62(2.68)$ & 0.8 & 0.43 \\
\hline HDL & $47.81(11.66)$ & $47.37(8.79)$ & 0.16 & 0.88 \\
\hline LDL & $120.15(26.18)$ & $123.33(36.12)$ & -3.71 & 0.71 \\
\hline
\end{tabular}

Abbreviation: SD, standard deviation; WHR, waist-hip ratio; BMI, Body Mass Index; LDL, Low-Density Lipoprotein; HDL, High-Density Lipoprotein. 


\subsection{The Changes of the Pretest to Posttest in Each Group (Within-Groups)}

Table 2 and 3 portrays the study variable changes before and after 12 weeks of the three variables follow-ups by paired-test in the experimental and control groups. The intervention effect estimates revealed no significant changes in WHR, BMI, HDL and LDL in the experimental group (see Table 2). However, in the control group, the posttest WHR was higher than the pretest and the posttest LDL was lower than the pretest $(P<0.001)$. (See Table 2).

Table 2. Comparisons of changes in WHR, BMI, LDL and HDL for pretest within each group (within groups) ( $N$ $=54)$

\begin{tabular}{|c|c|c|c|c|c|c|c|c|}
\hline Items & Experimental & oup $(N=27)$ & & & Control gro & $(N=27)$ & & \\
\hline & Pretest & Posttest & $t$ & $p$ & Posttest & Pretest & $t$ & $p$ \\
\hline WHR & $0.855(0.415)$ & $0.857(0.429)$ & -0.49 & 0.64 & $0.85(0.36)$ & $\begin{array}{l}0.87 \\
(0.43)\end{array}$ & -3.95 & $0.001 * *$ \\
\hline BMI & $22.19(2.52)$ & $21.97(2.49)$ & 2.01 & 0.55 & $\begin{array}{l}21.62 \\
(2.68)\end{array}$ & $\begin{array}{l}21.84 \\
(2.75)\end{array}$ & -1.95 & 0.069 \\
\hline HDL & $47.81(11.66)$ & $51.26(11.34)$ & -1.61 & 0.12 & $\begin{array}{l}47.37 \\
(8.79)\end{array}$ & $\begin{array}{l}45.19 \\
(9.66)\end{array}$ & 1.68 & 0.104 \\
\hline LDL & $\begin{array}{l}120.15 \\
(26.18)\end{array}$ & $117.15(23.62)$ & 0.98 & 0.34 & $\begin{array}{l}123.33 \\
(36.12)\end{array}$ & $\begin{array}{l}110.70 \\
(36.96)\end{array}$ & 4.12 & $0.000^{* *}$ \\
\hline
\end{tabular}

$* p<.05 * * p<.001$

Abbreviation: SD, standard deviation; WHR, waist-hip ratio; BMI, Body Mass Index; LDL, Low-Density Lipoprotein; HDL, High-Density Lipoprotein.

\subsection{The Comparison of the Changes of WHR, BMI, HDL and LDL (Pretest to Posttest) Between Two Groups}

The comparison of the changes of WHR, BMI, HDL and LDL in the pretest and posttest of two groups showed statistical significance. See Table 4. $(t=-2.76-9-2.258 ; P<0.05)$. The experimental group showed the more improved BMI, HDL and LDL after 12 weeks than the pretest, but the WHR increased slightly in the posttest, however in the control group WHR showed more increasing than the experimental group after 12 weeks; both BMI and HDL showed worse performance after 12 weeks in the control group than in the experimental group. Only LDL showed more improvement after 12 weeks compared with the experimental group.

Table 3. Comparisons of changes in WHR, BMI, LDL and HDL between the intervention and the controlled groups (between groups) $(N=54)$

\begin{tabular}{lllll}
\hline Items & Experimental $(N=27)$ & Control $(N=27)$ & $\mathrm{t}$ & $\mathrm{p}$ \\
\hline MHR & $0.002(0.02)$ & Mean $(S D)$ & & \\
\hline BMI & $-0.22(0.56)$ & $0.013(0.02)$ & -2.59 & $0.013^{*}$ \\
\hline HDL & $3.44(11.12)$ & $0.22(0.59)$ & -2.76 & $0.008^{*}$ \\
\hline LDL & $-3.00(15.90)$ & $-2.19(6.74)$ & 2.25 & $0.029^{*}$ \\
\hline
\end{tabular}

$* p<.05 * * p<.001$

Abbreviation: SD, standard deviation; WHR, waist-hip ratio; BMI, Body Mass Index; LDL, Low-Density Lipoprotein; HDL, High-Density Lipoprotein.

\section{Discussion}

When comparing the changes of the pretest and the posttest in WHR and BMI between the two groups, there is a 
statistical difference. After 12 weeks of exercise in Baduanjin, the experimental group has a downward trend, but the control group has an increasing trend with time.

Therefore, regular exercise is recommended for menopausal women to control weight. The waist-hip ratio ( 0.85 $\pm 0.386)$ and BMI $(21.90 \pm .2 .6)$ of the average age of the patients in this study were lower than 1,234 women with menopause in China, with an average age of 55 (50-61). WHR: 0.85, which may be related to the younger age group in this study. WHO in 2011, demonstrated that waist-hip changes with age or diet, and height changes are relatively small, the waist-to-height ratio, WHR is recommended to be added as an evaluation indicator (WHO, 2011), and in systematic review researches for body mass index (BMI), a total of 16 literatures including waist circumference (WC) and WHR were found. Five literatures confirmed the method of confirming the effectiveness in the elderly population. Comprehensive analysis of statistical results determined the best anthropometric index for waist-to-height ratio (WHR) to evaluate non-disease-related obesity. (Corrêa, Thumé, De Oliveira \& Tomasi, 2016). Visceral obesity, will increase the risk of cardiovascular disease and hypertension, and a closer relationship between various obesity-related metabolic diseases, such as the risk of hypertension, diabetes, hyperlipidemia, and cardiovascular disease (Liu, Ma, Lou \& Zhu, 2014), so it has been confirmed that menopausal women should pay attention to the changes in waist-to-hip and waist to height ratio and emphasize the prevention of chronic diseases. Future research can add WHR as a reference for further discussion (Liu, Ma, Lou, \& Zhu, 2014). Therefore, it has been confirmed that menopausal women should pay attention to the changes in waist-to-hip and waist to height ratio and emphasize the prevention of chronic diseases. Future research can add WHR as a reference for further discussion (Liu, Ma, Lou, \& Zhu, 2014)

Baduanjin exercise can improve WHR. It may be becuase Baduanjin exercise is a type of Qigong exercise (Wang \& Zhang, 2015; Wikipedia, 2019). Baduanjin exercise uses natural energy to balance the body and breathing through eight steps of simple exercises. The coordination of energy and thought is a gentle and safe aerobic exercise (Li et al, 2014; Ying, Min, Lei, Na, Li \& Jing, 2019), so it is necessary to take a deep-breathing method of Qixia Dantian (respiratory maneuver of intra-abdominal pressure) to achieve Qigong and aerobic effects (more than four types of movements including deep squat). Squats are one of the exercises for core muscles, so they increase the strength of core muscles, with reduced WHR too.

Dynamic squat is a closed dynamic chain exercise (Escamilla, Fleisig, Zheng, Barrentine, Wilk \& Andrews, 1998). It is one of the most widely used and effective resistance training to strengthen the lower limbs, preventing injuries and improving athletic performance (Hartmann, Wirth \& Klusemann, 2013) involved the body's largest and strongest muscles (quadriceps, gluteus maximus, and erector spinae, etc.), requiring coordinated movements of the spine, hips, knees, and ankles. It is beneficial to many functional activities and sports, often used in rehabilitation treatment of many chronic diseases (Schoenfeld, 2010), such as chronic obstructive pulmonary disease, stroke, and cerebral palsy (Garvey et al., 2016; Gray, Ivanova \& Garland, 2012; Eken, Harlaar, Dallmeijer, de Waard, van Bennekom, \& Houdijk, 2017). Squats have been proven to be safe movements for the musculoskeletal system (Kianifar, Lee, Raina \& Kulić, 2017; Simao et al., 2012). It can be deduced that Baduanjin exercise can reduce WHR and MBI due to the core exercise of squat and the benefits of aerobic exercise.

HDL and LDL: The women of this study revealed higher LDL $(121.741 \pm 31.290 \mathrm{mg} / \mathrm{dl})$ and lower HDL (47.593 $\pm 10.231 \mathrm{mg} / \mathrm{dl}$ ) than the guidelines for dyslipidemia of the European Society of Cardiology (2020) - the new standard of blood lipids, and LDL which recommended as high-risk. The goal of primary prevention of cardiovascular diseases in menopausal women should maintain LDL below $100 \mathrm{mg} / \mathrm{dl}$ and increase HDL to above $50 \mathrm{mg} / \mathrm{dl}$ (Mach et al., 2020). The dietary pattern in $70.4 \%$ of the women in the current study were mostly vegetarian. However, the dietary content of vegetarians is still to be discussed. Women in the control group showed that LDL of the posttest decreased significantly. It may be a Hawthorne effect. The women in the control group knew that they were being observed, and decided to make dietary adjustments which needs to be further explored in future research. Therefore, future research design can be aimed at diet control and exercise training. Three groups including the diet and exercise training groups are to be analyzed for the benefits in improving blood lipid concentration.

People cannot guarantee to lower LDL by eating as a vegetarian, the content of food is more important than just vegetarians. While static lifestyle habits will increase high-density cholesterol, so it is necessary to strengthen exercise to increase high-density cholesterol and reduce the incidence of chronic cardiovascular disease. It can be seen that the subjects of this study were in great need of increasing high-density cholesterol through exercise to reduce the incidence of chronic diseases.

Perimenopause is the key period for maintaining health management; it is also the best period for Baduanjin sports intervention nursing measures to reduce the prevalence of hypertension, hyperglycemia, and 
hyperlipidemia. The effects of twelve Baduanjin regular exercises demonstrated similar result as the previous Qigong study with the same effect of exercise training on the blood lipid concentration in patients with mild hypertension (Li, Wu, Jin, \& Pan, 2019).

In the current study, exercise effects of the experimental group for practicing 12 weeks of Baduanjin regularly can maintain the waist-hip ratio and BMI; while the control group without exercise would increase their WHR and BMI after 12 weeks. The results are similar to previous study; Wen, et al. (2017) used a systematic review of randomized controlled trials and meta-analysis to explore the benefits of Baduanjin exercise for patients with type 2 diabetes, and found that blood pressure, weight, blood glucose, and LDL were significantly reduced compared to non-intervention Baduanjin measures. (Xiong, Wang, Li, Zhang \& Li, 2015).

\section{Conclusion}

\subsection{Conclusion}

In conclusion, doing Baduanjin exercise of 10-15 minutes twice per day at home for twelve weeks had positive effects in in decreasing BMI, increasing HDL, decreasing LDL and more slowly increasing WHR compared with the control group. The current study demonstrated that Baduanjin home exercise would through improving women's hyperlipidemia and BMI to health promotion for reducing CVD risk factors. It is suggested that Baduanjin home exercise would be a good choice for busy menopausal women to do regular exercise without the limitation of time, location or peer.

\subsection{Recommendation}

It is crucial to establish a Baduanjin exercise program for mid-life women in community to empower their self-care for health promotion ability. Instead of emphasizing menopause as an increasing CVD mobility, we should provide more western medicine integrated with traditional-medicine health promotion program for

greeting the coming of healthy menopause and aging from multidimensional perspectives including body-mind changes, culture, self-care, and easy home exercises.

\subsection{Limitation}

The study design used non-random assignment of sampling to facilitate sampling, so the effect of causality is weak. Moreover, due to regional differences, deviations in external validity cannot be inferred to other counties and cities, and future research needs to include more samples from different regions and long-term follow-up study.

In addition, how to incentive for women to keep on this home-based exercise is the challenge for future study

\section{References}

Baghbani-Oskouei, A., Tohidi, M., Asgari, S., Ramezankhani, A., Azizi, F., \& Hadaegh, F. (2018). Serum Lipids During 20 Years in the Tehran Lipid and Glucose Study: Prevalence, Trends and Impact on Non-Communicable Diseases. International Journal Endocrinology Metabolism, 16(4 Suppl), e84750. https://doi.org/10.5812/ijem.84750

Borg, G. V. (1982). Psychophysical bases of perceived exertion. Medicine and Science in Sports and Exercise, 14(5), 337-381.

Cengiz, H., Kaya, C., Suzen, C. S., \& Alay, I. (2019). The relationship between menopausal symptoms and metabolic syndrome in postmenopausal women. Journal Obstetrical Gynaecology, 39(4), 529-533. https://doi.org/10.1080/01443615.2018.1534812

Colpani, V., Baena, C. P., Jaspers, L., Van Dijk, G. M., \& Franco, O. H. (2018). Lifestyle factors, cardiovascular disease and all-cause mortality in middle-aged and elderly women: a systematic review and meta-analysis. European Journal Epidemiology, 33(9), 831-845. https://doi.org/10.1007/s10654-018-0374-z

Corrêa, M. M., Thumé, E., De Oliveira, E. R., \& Tomasi, E. (2016). Performance of the waist-to-height ratio in identifying obesity and predicting non-communicable diseases in the elderly population: A systematic literature review. Archives Gerontology Geriatric, 65, 174-82. https://doi.org/10.1016/j.archger.2016.03.021

Dmitruk, A., Czeczelewski, J., Czeczelewska, E., Golach, J., \& Parnicka, U. (2018). Body composition and fatty tissue distribution in women with various menstrual.

Eken, M. M., Harlaar, J., Dallmeijer, A., de Waard, E., van Bennekom, C. M., \& Houdijk, H. (2017). Squat test performance and execution in children withand without cerebral palsy. Clinical Biomechanics, 41, 98-105. https://doi.org/10.1016/j.clinbiomech.2016.12.006 
Escamilla, R. F., Fleisig, G. S., Zheng, N., Barrentine, S. W., Wilk, K. E., \& Andrews, J. R. (1998). Biomechanics of the knee during closed kinetic chain and open kinetic chain exercises. Medicine Science Sports Exercise, 30, 556-569.

Ford, E. S., Zhao, G., Tsai, J., \& Li, C. (2011). Low-risk lifestyle behaviors and all-cause mortality: findings from the National Health and Nutrition Examination Survey III Mortality Study. American of Journal Public Health, 101(10), 1922-9. https://doi.org/10.2105//ajph.2011.300167

Garvey, C., Bayles, M. P., Hamm, L. F., Hill, K., Holland, A., Limberg, T. M., \& Spruit, M. A. (2016). Pulmonary Rehabilitation Exercise Prescription in Chronic Obstructive Pulmonary Disease: Review of Selected Guidelines: An official statement from the American association of cardiovascular and pulmonary rehabilitation. Journal of Cardiopulmonary Rehabilitation Prevent, 36(2), 75-83. https://doi.org/10.1097/HCR.0000000000000171

Gray, V. L., Ivanova, T. D., \& Garland, S. J. (2012). Control of fast squatting movements after stroke. Clinical Neurophysiology, 123(2), 344-350. https://doi.org/10.1016/j.clinph.2011.07.003

Hartmann, H., Wirth, K., \& Klusemann, M. (2013). Analysis of the load on the knee joint and vertebral column with changes in squatting depth and weight load. Sports Medicine, 43, 993-1008. https://doi.org/10.1007/s40279-013-0073-6

Hashemi Nazari, S. S., Shakiba, M., Khalili, D., Hadaegh, F., Tohidi, M., \& Azizi, F. (2015). High-density lipoprotein cholesterol, a protective or a risk factor for developing coronary heart disease? Tehran lipid and glucose study. Journal of Clinical Lipidology, 9(4), 553-8. https://doi.org/10.1016/j.jacl.2015.04.001

Hunter, M. S., Hardy, C., Norton, S., \& Griffiths, A. (2006). Study protocol of a multicentre randomised controlled trial of self-help cognitive behaviour therapy for working women with menopausal symptoms. Maturitas, 92, 186-92.

Kianifar, R., Lee, A., Raina, S., \& Kulic, D. (2017). Automated assessment of dynamic knee valgus and risk of knee injury during the single leg squat. IEEE Journal of Translational Engineering in Health Medicine, 14, 5. https://doi.org/10.1109/JTEHM.2017.2736559

Korsager Larsen, M., \& Matchkov, V. V. (2016). Hypertension and physical exercise: The role of oxidative stress. Medicina (Kaunas), 52(1), 19-27.

Li, H., Ge, D., Liu, S., Zhang, W., Wang, J., Si, J., \& Zhai, J. (2019). Baduanjin exercise for low back pain: A systematic review and meta-analysis. Complementary Therapies in Medicine, 43, 109-116. https://doi.org/10.1016/j.ctim.2019.01.021

Li, R., Jin, L., Hong, P., He, Z. H., Huang, C. Y., Zhao, J. X., Wang, M., \& Tian, Y. (2014). The effect of baduanjin on promoting the physical fitness and health of adults. Evidence- Based Complementary and Alternation Medicine. https://doi.org/10.1155/2014/784059

Li, W., Wu, Z., Jin, C., \& Pan, H. (2019). Clinical Observation on Effect of Baduanjin on Blood Glucose and Blood Pressure in Patients with Impaired Glucose Regulation Complicated with Mild Hypertension. Journal of New Chinese Medicine, 51, 7.

Lin, Y. Y., \& Lee, S. D. (2018). Cardiovascular Benefits of Exercise Training in Postmenopausal Hypertension. International Journal Molecular Science, 19(9), 2523. https://doi.org/10.3390/ijms19092523

Liu, P., Ma, F., Lou, H., \& Zhu, Y. (2014). Utility of obesity indices in screening Chinese postmenopausal $\begin{array}{llll}\text { women for metabolic } & \text { Menopause, 21(5), 509-514. }\end{array}$ https://doi.org/10.1097/GME.0b013e3182a170be

Mach, F., Baigent, C., Catapano, A. L., Koskinas, K. C., Casula, M., Badimon, L., \& Wiklund, O. (2020). 2019 ESC/EAS Guidelines for the management of dyslipidaemias: lipid modification to reduce cardiovascular risk: The Task Force for the management of dyslipidaemias of the European Society of Cardiology (ESC) and European Atherosclerosis Society (EAS). European Heart Journal, 41(1), 111-188. https://doi.org/10.1093/eurheartj/ehz455

McSweeney, J. C., Rosenfeld, A. G., Abel, W. M., Braun, L. T., Burke, L. E., Daugherty, S. L., \& Reckelhoff, J. F. (2016). Preventing and Experiencing Ischemic Heart Disease as a Woman: State of the Science: A Scientific Statement from the American Heart Association. Circulation, 133(13), 1302-31. https://doi.org/10.1161/CIR.0000000000000381

Ministry of Health and Welfare, Taiwan, ROC. (2019a). Investigation and Analysis of Women's Waist 
Circumference and Three High Diseases after Menopause report. Retrieved from https://www.hpa.gov.tw/Pages/Detail.aspx?nodeid=3804\&pid=10454

Ministry of Health and Welfare, Taiwan, ROC. (2019b). Prevention of chronic disease of women. Retrieved from https://www.hpa.gov.tw/Pages/Detail.aspx?nodeid=3804\&pid=10454

Ministry of Health and Welfare, Taiwan, ROC. (2019c). Women's Menopause Health Handbook. Retrieved from https://www.hpa.gov.tw/Pages/EBook.aspx?nodeid=1148

Muka, T., Oliver-Williams, C., Kunutsor, S., Laven, J. S., Fauser, B. C., Chowdhury, R., Kavousi, M., \& Franco, O. H. (2016). Association of Age at Onset of Menopause and Time Since Onset of Menopause with Cardiovascular Outcomes, Intermediate Vascular Traits, and All-Cause Mortality: A Systematic Review and Meta-analysis. JAMA Cardiology, 1(7), 767-776. https://doi.org/10.1001/jamacardio.2016.2415

Mumusoglu, S., \& Yildiz, B. O. (2019). Metabolic syndrome during Menopause. Curr Vasc Pharmacol, 17(6), 595-603. https://doi.org/10.2174/1570161116666180904094149

Nejat, A., Mirbolouk, M., Mohebi, R., Hasheminia, M., Tohidi, M., Saadat, N., Azizi, F., \& Hadaegh, F. (2014). Changes in lipid measures and incident coronary heart disease: Tehran lipid and glucose study. Clinical Biochemistry, 47(13-14), 1239-44. https://doi.org/10.1016/j.clinbiochem.2014.03.004

Pal, S., Radavelli-Bagatini, S., \& Ho, S. (2013). Potential benefits of exercise on blood pressure and vascular function. Journal of American Social Hypertension, 7(6), 494-506.

Palacios, S., Henderson, V., Siseles, N., Tan, D., \& Villaseca, P. (2010). Age of menopause and impact of climacteric symptoms by geographical region. Climacteric: The Journal of the International Menopause Society, 13, 419-28. https://doi.org/10.3109/13697137.2010.507886

Rago, V., Brito, J., Figueiredo, P., Costa, J., Krustrup, P., \& Rebelo, A. (2019). Internal training load monitoring in professional football: a systematic review of methods using rating of perceived exertion. The Journal of Sports Medicine and Physical Fitness, 24. https://doi.org/10.23736/s0022-4707.19.10000-x

Schoenfeld, B. J. (2010). Squatting kinematics and kinetics and their application to exercise performance. Journal Strength Conducive Research, 24, 3497-3506.

Simão, A. P., Avelar, N. C., Tossige-Gomes, R., Neves, C. D., Mendonça, V. A., Miranda, A. S., \& Lacerda, A. C. (2012). Functional performance and inflammatory cytokines after squat exercises and whole-body vibration in elderly individuals with knee osteoarthritis. Archives. Physical. Medicine. Rehabilitation, 93(10), 1692-1700. https://doi.org/10.1016/j.apmr.2012.04.017

Tabatabaei-Malazy, O., Djalalinia, S., Asayesh, H., Shakori, Y., Esmaeili Abdar, M., \& Qorbani, M. (2018). Menopause and metabolic syndrome in the Middle East countries; a systematic review and meta-analysis study. Journal of Diabetes Metabolic Disorder, 17(2), 357-364. https://doi.org/10.1007/s40200-018-0375-1

Wang, C. Y., \& Zhang, H. (2015). Influence of Baduanjin combined with routine treatment on blood glucose level in type 2 diabetic patients. China Medicine Pharm Ology, 22, 49-52.

Wang, N., Qin, M. Z., \& Cui, J. (2016). Lipid Profile Comparison between Pre- and Post-Menopausal Women. $\begin{array}{llllll}\text { Zhonghua Xin Xue Guan Bing } Z a \text { Zhi, 44(9), } & \text { 799-804. }\end{array}$ https://doi.org/10.3760/cma.j.issn.0253-3758.2016.09.013

Wen, J., Lin, T., Cai, Y., Chen, Q., Chen, Y., Ren, Y., \& Wu, W. (2017). Baduanjin Exercise for Type 2 Diabetes Mellitus: A Systematic Review and Meta-Analysis of Randomized Controlled Trials. Evidence- Based Complementary and Alternation Medicine. https://doi.org/10.1155/2017/8378219

Wikipedia. (2019). Baguanjin Qigong. Retrieved January 18, 2019, from https://en.wikipedia.org/wiki/Baduanjin_qigong

World Health Organization. (2011). Waist circumference and waist-hip ratio: report of a WHO expert consultation, Geneva. Retrieved from https://apps.who.int/iris/handle/10665/44583

Xiao, C. M., \& Zhuang, Y. C. (2015). Effect of health Baduanjin Qigong for mild to moderate Parkinson's disease. Geriatric Gerontology International, 16, 911.

Xiao, X., Wang, J., Gu, Y., Cai, Y., \& Ma, L. (2018). Effect of community-based practice of Baduanjin on self-efficacy of adults with cardiovascular diseases. PLoS One, 13(7), e0200246. https://doi.org/10.1371/journal.pone.0200246. eCollection 2018

Xiong, X., Wang, P., Li, S., Zhang, Y., \& Li, X. (2015). Effect of Baduanjin exercise for hypertension: a 
systematic review and meta-analysis of randomized controlled trials. Maturitas, 80(4), 370-8. https://doi.org/10.1016/j.maturitas.2015.01.002

Ying, W., Min, Q. W., Lei, T., Na, Z. X., Li, L., \& Jing, L. (2019). The health effects of Baduanjin exercise (a type of Qigong exercise) in breast cancer survivors: A randomized, controlled, single-blinded trial. European Journal of Oncology Nursing, 39, 90-97. https://doi.org/10.1016/j.ejon.2019.01.007

\section{Copyrights}

Copyright for this article is retained by the author(s), with first publication rights granted to the journal.

This is an open-access article distributed under the terms and conditions of the Creative Commons Attribution license (http://creativecommons.org/licenses/by/4.0/). 